OPEN ACCESS

Edited by:

Yunkai Zhang,

Vanderbilt University Medical Center,

United States

Reviewed by:

Chuan Wang,

Auburn University, United States

Shujue Lan,

Institute of Biochemistry and Cell

Biology, Shanghai Institutes for

Biological Sciences (CAS), China

Jing Zhao,

Fourth Military Medical University,

China

*Correspondence:

Zhi Shi

tshizhi@jnu.edu.cn Xiao-Shun $\mathrm{He}$

gdtrc@163.com

Qi Zhou

zhouqi@mail.sysu.edu.cn

tThese authors have contributed equally to this work

Specialty section:

This article was submitted to Cancer Molecular Targets and

Therapeutics,

a section of the journal

Frontiers in Oncology

Received: 25 January 2019

Accepted: 22 February 2019

Published: 15 March 2019

Citation:

Huang $S-Z$, Wei M-N, Huang J-R, Zhang Z-J, Zhang W-J, Jiang Q-W, Yang $Y$, Wang $H-Y$, Jin $H-L$, Wang $K$, Xing Z-H, Yuan M-L, Li Y, He X-S, Shi Z and Zhou Q (2019) Targeting

TF-AKT/ERK-EGFR Pathway

Suppresses the Growth of

Hepatocellular Carcinoma.

Front. Oncol. 9:150.

doi: 10.3389/fonc.2019.00150

\title{
Targeting TF-AKT/ERK-EGFR Pathway Suppresses the Growth of Hepatocellular Carcinoma
}

\begin{abstract}
Shan-Zhou Huang ${ }^{1,2 \dagger}$, Meng-Ning Wei ${ }^{3 \dagger}$, Jia-Rong Huang ${ }^{3 \dagger}$, Zi-Jian Zhang ${ }^{4}$, Wen-Ji Zhang ${ }^{3}$, Qi-Wei Jiang ${ }^{3}$, Yang Yang ${ }^{3}$, Huan-Yu Wang ${ }^{5}$, Hui-Lin Jin ${ }^{1}$, Kun Wang ${ }^{3}$, Zi-Hao Xing ${ }^{3}$, Meng-Ling Yuan ${ }^{3}$, Yao $\mathrm{Li}^{3}$, Xiao-Shun $\mathrm{He}^{2 *}$, Zhi Shi ${ }^{2 *}$ and Qi Zhou ${ }^{1,6 *}$
\end{abstract}

${ }^{1}$ Department of Hepatic Surgery, The First Affiliated Hospital, Sun Yat-sen University, Guangzhou, China, ${ }^{2}$ Organ Transplant Center, The First Affiliated Hospital, Sun Yat-sen University, Guangzhou, China, ${ }^{3}$ Guangdong Provincial Key Laboratory of Bioengineering Medicine, Department of Cell Biology and Institute of Biomedicine, National Engineering Research Center of Genetic Medicine, College of Life Science and Technology, Jinan University, Guangzhou, China, ${ }^{4}$ Department of Hepatobiliary Surgery, The Seventh Affiliated Hospital of Sun Yat-sen University, Shenzhen, China, ${ }^{5}$ Department of Thyroid and Breast Surgery, Nanshan District People's Hospital, Shenzhen, China, ${ }^{6}$ Department of General Surgery, Hui Ya Hospital of The First Affiliated Hospital, Sun Yat-sen University, Huizhou, China

Tissue factor (TF) is a transmembrane glycoprotein to initiate blood coagulation and frequently overexpressed in a variety of tumors. Our previous study has showed that the expression of TF is upregulated and correlated with prognosis in hepatocellular carcinoma (HCC). However, the role and molecular mechanism of TF in the growth of $\mathrm{HCC}$ are still unclear. In vitro and in vivo functional experiments were performed to determine the effect of TF on the growth of HCC cells. A panel of biochemical assays was used to elucidate the underlying mechanisms. TF could promote the growth of HCC in vitro and in vivo by activating both ERK and AKT signaling pathways. TF induced EGFR upregualtion, and inhibition of EGFR suppressed TF-mediated HCC growth. In addition, TF protein expression was correlated with EGFR in HCC tissues. TF promotes HCC growth by upregulation of EGFR, and TF as well as EGFR may be potential therapeutic targets of HCC.

Keywords: hepatocellular carcinoma, tissue factor, epidermal growth factor receptor, AKT/ERK, tumor growth

\section{INTRODUCTION}

Hepatocellular carcinoma (HCC) is the fifth most lethal cancers worldwide, while China accounted for more than half of all cases and deaths in 2012 (1). More than 400,000 people die from liver cancer and over 450,000 new cases are diagnosed in China each year (2). Though the treatments for HCC have been greatly advanced in recent years, the outcome of HCC is still unoptimistic. Postoperative recurrence, the main reason for poor survival of HCC patients, mainly owes to the tendency of the invasion and metastasis of HCC cells $(3,4)$. Therefore, understanding the mechanism of HCC tumorigenesis and progression is critical to improve the clinical outcome of HCC patients.

Tissue factor (TF, also known as platelet tissue factor, factor III, thromboplastin, or CD142, encoded by the F3 gene) is a $47 \mathrm{kD}$ transmembrane glycoprotein that contains 263 amino acid residues totally including a 219 amino acid extracellular region, a 23 amino acid hydrophobic transmembrane region, and a 21 amino acids C-terminal intracellular tail (5). Originally, TF is found on the surface of intravascular cells, such as platelets, leukocytes, and endothelial cells and 
functions as the principal initiator of the extrinsic coagulation cascade by binding with circulating factor VII or VII $\alpha$ $(\mathrm{FVII} / \mathrm{VII} \alpha)$ (6). Recently, TF is frequently overexpressed in a variety of tumors, including breast cancer, colorectal carcinoma, gastric cancer, non-small cell lung, and pancreatic ductal carcinoma, etc. (7). We and other groups have reported that the expression of TF is upregulated and correlated with prognosis in HCC (8-10). In the current study, we investigate the role and molecular mechanism of TF in the growth of HCC cells.

\section{MATERIALS AND METHODS}

\section{Patients and Tissue Specimens}

A total 144 HCC tissues were obtained from patients who underwent curative resection between Jan 2008 and Dec 2010 at the First Affiliated Hospital, Sun Yat-sen University. None of the patients received neoadjuvant radiotherapy or chemotherapy before surgery. Signed informed consents were obtained from all patients. The study was approved by the ethics committee of the First Affiliated Hospital, Sun Yat-sen University.

\section{Cell Culture and Reagents}

The human HCC cell lines HepG2, BEL-7402, SK-HEP1, SMMC-7721, and normal hepatic cell line LO2 were from China Center for Type Culture Collection and cultured in Dulbecco's modified Eagle's medium (DMEM) supplemented with $10 \%$ fetal bovine serum (FBS), penicillin $(100 \mathrm{U} / \mathrm{ml})$ and streptomycin $(100 \mathrm{ng} / \mathrm{ml})$ in a humidified incubator at $37^{\circ} \mathrm{C}$ with 5\% $\mathrm{CO}_{2}$ atmosphere. U0126, LY294002, and Gefitinib were from ApexBio. Anti-TF (ab17375) and Anti-Ki-67 (2724-1) were from Abcam. Anti-pAKT (4060), Anti-AKT (4691), Anti-pERK (4370), and Anti-ERK (4695) antibodies were from Cell Signaling Technologies. Anti-EGFR (SC-03) and Anti-c-Myc (SC-40) antibodies were from Santa Cruz Biotechnology. Anti- $\beta$-actin
A
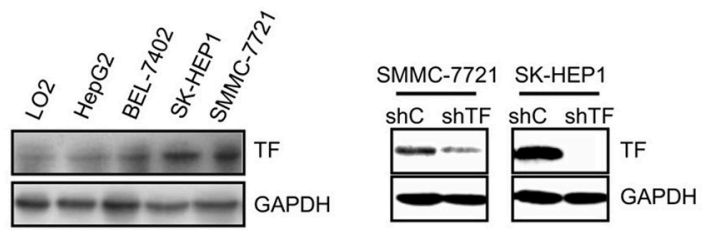

C

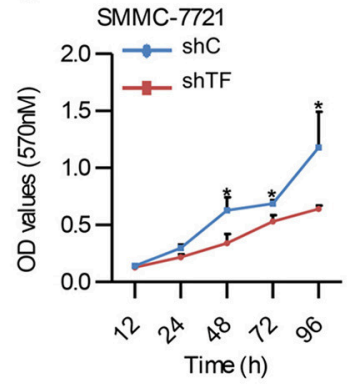

E

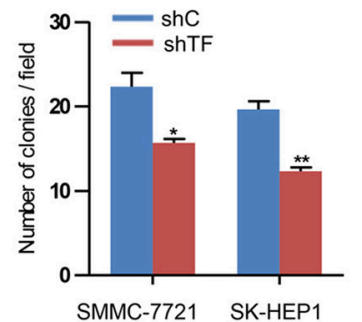

G

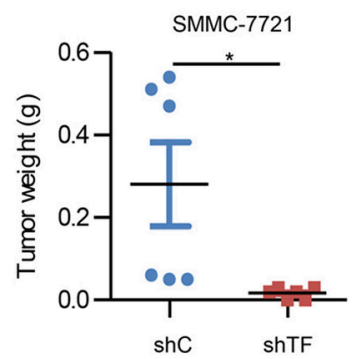

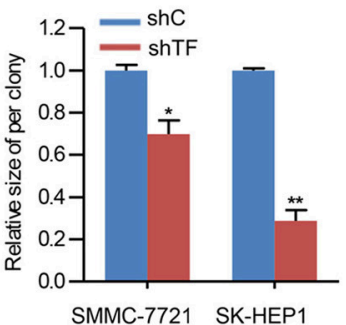

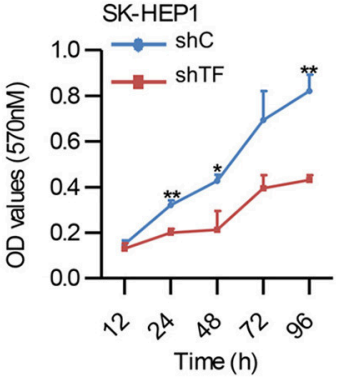

SMMC-7721 SK-HEP1

H

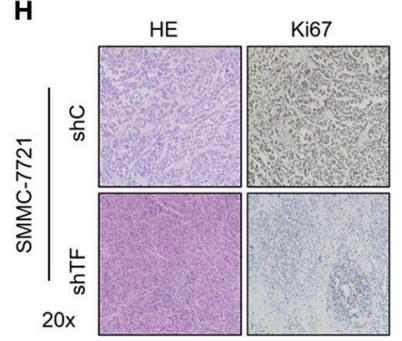

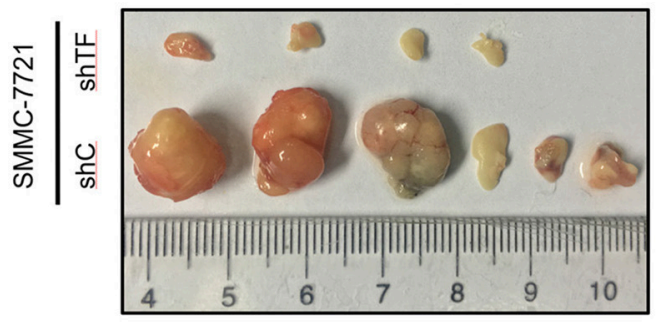

FIGURE 1 | Knockdown of TF inhibits the growth of HCC. (A,B) Western blot analysis of the protein expressions in the indicated cells. (C) Cell growth of the indicated cells as determined with MTT assay. (D) Representative images and (E) quantification of the indicated cells sphere as determined with sphere formation assay. (F) The indicated subcutaneous tumors and (G) tumor weight of nude mice were shown. (H) Representative images of H\&E and Ki-67 staining in the indicated tumor sections as determined with $\mathrm{IHC}$ assay. Error bars, mean $\pm \mathrm{SD}$. ${ }^{*} p<0.05$ and ${ }^{* *} p<0.01$ [two-tailed Student's $t$-test (C,E,G)]. 
(LK9001T) and Anti-GAPDH (LK9002T) antibodies were from Tianjin Sungene Biotech.

\section{Plasmid Construction and Lentivirus Production}

The human TF cDNA was cloned into pLVX-AcGFP1-N1 lentiviral vector, and shRNA targeting human TF mRNA ( $5^{\prime}$-GCGCUUCAGGCACUACAAA-3') was cloned into pLKO.1 lentiviral vector. Lentivirus was packaged in HEK293T cells and collected from the medium supernatant. Stable cell lines were established by infecting lentivirus into cells, followed by puromycin selection $(11,12)$.

\section{siRNA Transfection}

The EGFR siRNA (sense sequences: 5' - CUGACUCCGUCCAGUAUUGAU-3') and negative control siRNA were synthesized by Guangzhou Ribobio. Each siRNA solution was mixed gently with the respective volume of the X-tremeGENE siRNA Transfection Reagent and allowed to form transfection mixture for $20 \mathrm{~min}$. Cells were cultured in 6-well plate with DMEM until 50\% of confluence and added with the transfection mixture for $24 \mathrm{~h}$ before the next experiment $(13,14)$.

\section{Western Blot}

Cells were harvested and washed twice with cold PBS, then resuspended and lysed in RIPA buffer ( $1 \%$ NP-40, 0.5\% sodium deoxycholate, $0.1 \%$ SDS, $10 \mathrm{ng} / \mathrm{ml}$ PMSF, $0.03 \%$ aprotinin, $1 \mu \mathrm{M}$ sodium orthovanadate) at $4{ }^{\circ} \mathrm{C}$ for $30 \mathrm{~min}$. Lysates were centrifuged for $10 \mathrm{~min}$ at $14,000 \times \mathrm{g}$ and supernatants were stored at $-80^{\circ} \mathrm{C}$ as whole cell extracts. Proteins were separated on $12 \%$ SDS-PAGE gels and transferred to polyvinylidene difluoride membranes. Membranes were blocked with 5\% BSA and incubated with the indicated primary antibodies. Corresponding horseradish peroxidase-conjugated secondary antibodies were used against each primary antibody. Signals were detected using
A

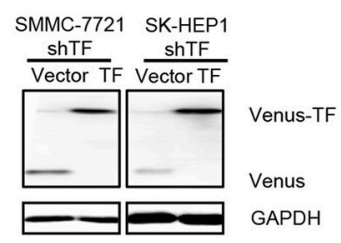

C

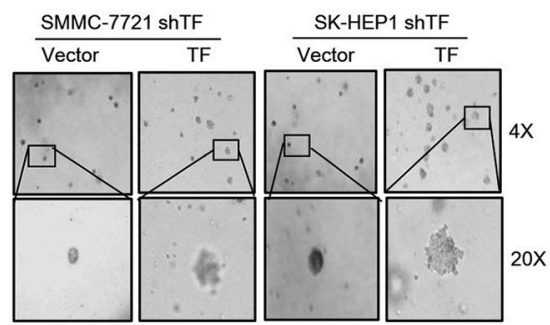

E

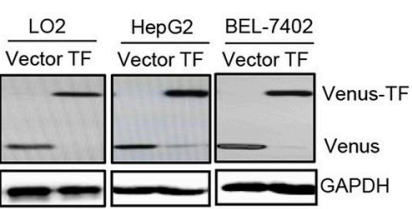

B
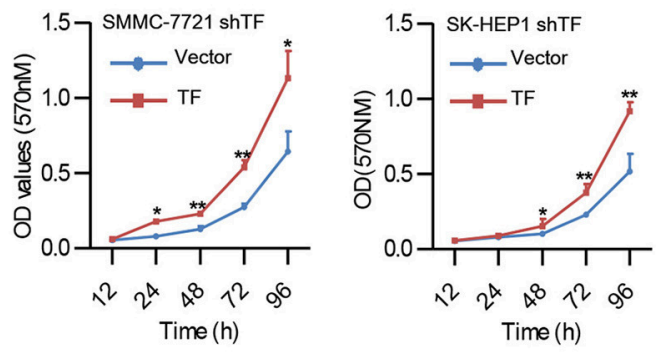

D
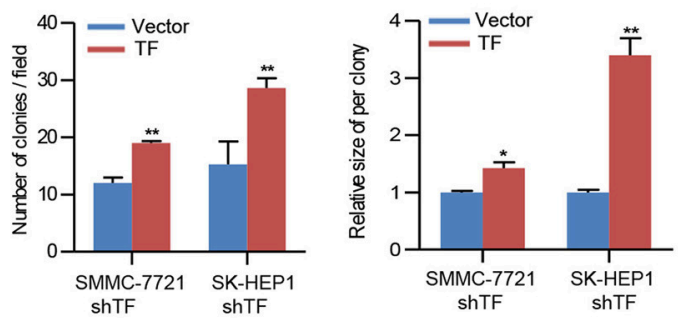

$\mathbf{F}$

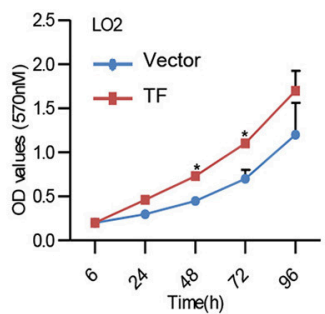

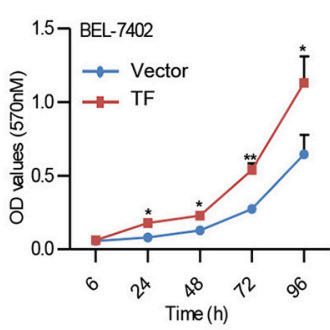

FIGURE 2 | Overexpression of TF promotes the growth of HCC. (A,E) Western blot analysis of the protein expressions in the indicated cells. (B,F) Cell growth of the indicated cells as determined with MTT assay. (C) Representative images and (D) quantification of the indicated cells sphere as determined with sphere formation assay. Error bars, mean \pm SD. ${ }^{*} p<0.05$ and ${ }^{* *} p<0.01$ [two-tailed Student's $t$-test (B,D,F)]. 
the ChemiDoc XRS chemiluminescent gel imaging system (BioRAD) $(15,16)$.

\section{MTT Assay}

Cells were seeded into a 96-well plate at a density of $0.5-1 \times 10^{4}$ cells/well and treated with various concentrations of agents. After 3 days, 3-(4, 5-dimethylthiazolyl-2)-2, 5diphenyltetrazolium bromide (MTT) was added to each well at a final concentration of $0.5 \mathrm{mg} / \mathrm{ml}$. After incubation for $4 \mathrm{~h}$, the medium and MTT solution were removed from each well, and formazan crystals were dissolved in $100 \mu \mathrm{l}$ of DMSO. Absorbance was measured at $570 \mathrm{~nm}$ by Multiscan Spectrum (Thermofisher) $(17,18)$.

\section{Sphere Formation Assay}

Cells were trypsinized, suspended in medium containing $0.3 \%$ agar and $10 \%$ FBS and seeded at a density of $5 \times 10^{2}$ cells/well in a 12-well plate. The agar-cell mixture was plated onto a bottom layer with $0.5 \%$ agar. Then treated cells were incubated in a humidified incubator and fresh medium was added every 3 days. Two weeks later, colonies were analyzed microscopically $(19,20)$.

\section{Nude Mice Xenograft Tumor Assay}

The female Balb/c nude mice with 5 weeks old and 16-18 g weight were obtained from the Shanghai SLAC Laboratory Animal Co and maintained with sterilized food and water. For xenograft tumor assay, $4 \times 10^{6}$ cells in $100 \mu \mathrm{l}$ of DMEM were injected subcutaneously under the shoulder of six mice per group. The mice were anesthetized after experiment, and tumors or lungs were removed, weighed, and sectioned. All experimental procedures were approved by the Institutional Animal Care and Use Committee of Jinan University $(21,22)$.

\section{Immunohistochemistry Assay}

Immunohistochemistry (IHC) assay was performed with a microwave-enhanced avidin-biotin staining method. Formalinfixed, paraffin embedded human HCC tissue array and subcutaneous tumors in mice were stained with antibodies, respectively, using a microwave-enhanced avidin-biotin staining method. To quantify the protein expression, the following formula was used: IHC score $=$ percentage of positive cells $\times$ intensity score. The intensity was scored as follows: 0 , negative (no staining); 1, weak (light yellow); 2, moderate (yellow brown); and 3 , intense (brown) $(23,24)$.

\section{Statistical Analysis}

Statistical analyses were performed using SPSS 19.0 for Windows (SPSS) and Graph-Pad Prism 6. Data were expressed as the mean \pm standard deviation (SD) from at least three independent experiments. Quantitative data between two groups were compared using the Student's $t$-test. Categorical data

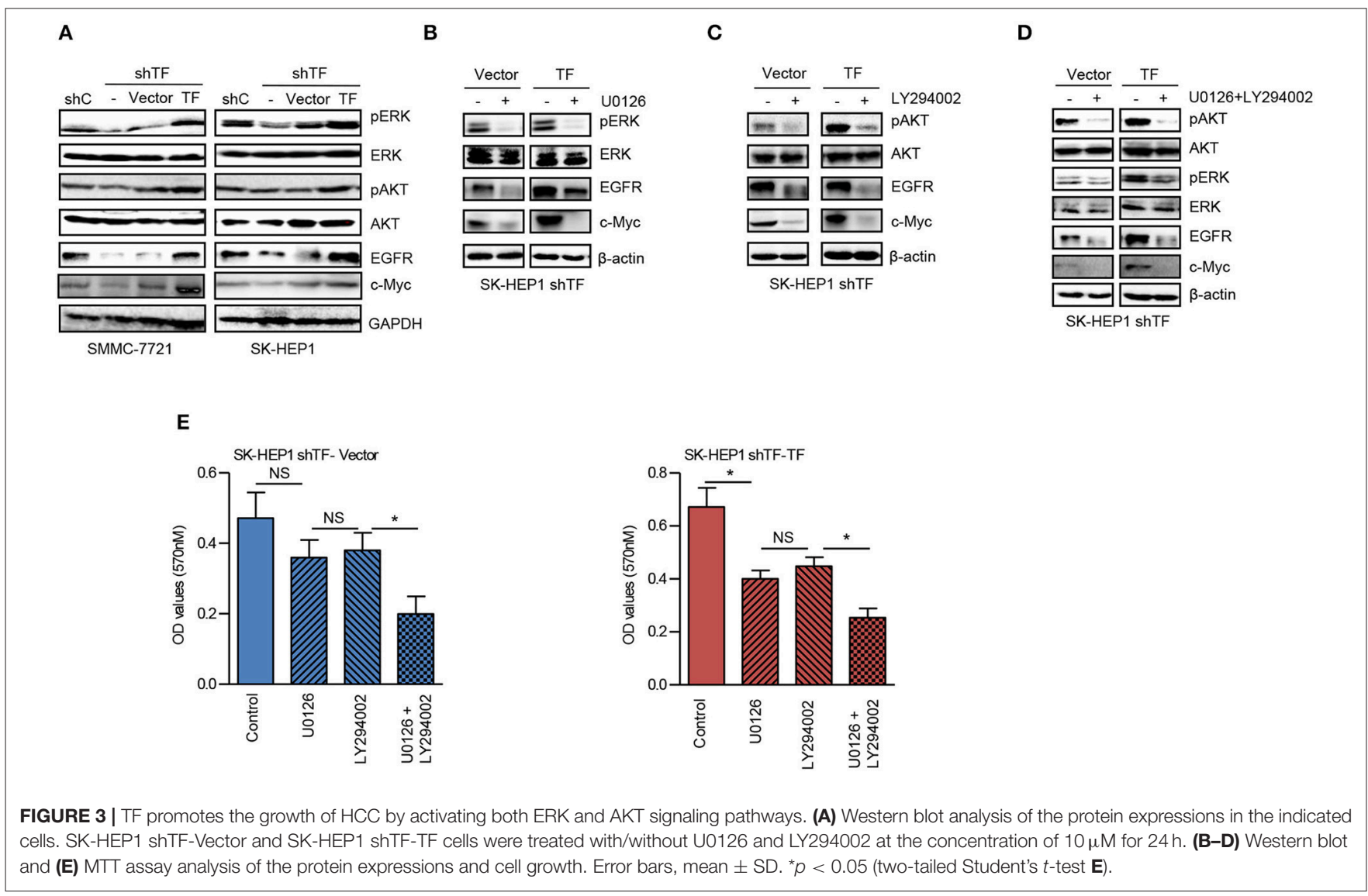


were analyzed by the $\chi^{2}$ test or Fisher exact test. Correlations between different protein expressions level were determined using Spearman's rank analysis. The $p<0.05$ was considered as statistical significance. ${ }^{*} p<0.05$; ${ }^{* *} p<0.01$; NS: no statistical significance.

\section{RESULTS}

\section{Knockdown of TF Inhibits the Growth of HCC}

To explore the potential biological function of TF in HCC, we first examined the protein expression of $\mathrm{TF}$ in human HCC cell lines including HepG2, BEL-7402, SK-HEP1, SMMC7721, and normal hepatic cell line LO2. Notably, all HCC cell lines displayed higher protein levels of TF than normal hepatic cell line, and SK-HEP1 and SMMC-7721 cells showed the highest protein levels of $\mathrm{TF}$ in all cells (Figure 1A). To further investigate the role of TF in HCC malignancy, we generated the cells with shRNA-mediated stable knockdown of endogenous TF in both SK-HEP1 and SMMC-7721 cells (Figure 1B). Knockdown of TF decreased the cell amounts, sphere numbers and sizes in both SK-HEP1 and SMMC7721 cells as detected by MTT and sphere formation assays (Figures 1C-E). Additionally, the data of subcutaneous tumor models in nude mice showed that TF knockdown inhibited the growth of SMMC-7721 xenografts by decreasing the volumes and weights of tumors as well as the numbers of $\mathrm{Ki}^{+} 7^{+}$proliferating cells (Figures 1F-H).

\section{Overexpression of TF Promotes the Growth of HCC}

To confirm the effect of TF on HCC growth, we performed rescue experiments by ectopic expression of $\mathrm{TF}$ in both TF-silenced SMMC-7721 and SK-HEP1 cells (Figure 2A). Ectopic expression of TF increased the cell amounts, sphere numbers, and sizes in both TF-silenced SMMC7721 and SK-HEP1 cells (Figures 2B-D). Furthermore, overexpression of TF increased the cell amounts in
LO2, HepG2, and BEL-7402 cells (Figures 2E, F). Taken together, these results suggest that $\mathrm{TF}$ can promote the growth of HCC.

\section{TF Promotes the Growth of HCC by Activating Both ERK and AKT Signaling Pathways}

To further explore the molecular mechanism of TF-promoted HCC growth, we detected the downstream signaling pathway of TF. As shown in Figure 3A, knockdown of TF decreased the protein levels of phosphorylated ERK (pERK), phosphorylated AKT (pAKT), and their downstream transcriptional factor cMyc in both SMMC-7721 and SK-HEP1 cells. While ectopic expression of TF increased the protein levels of pERK, pAKT and c-Myc in both TF-silenced SMMC-7721 and SK-HEP1 cells. Interesting, the protein level of EGFR was downregulated in TF-silenced HCC cells and upregulated in TF-overexpressed HCC cells (Figure 3A). To define the roles of ERK and AKT in TF-mediated HCC growth, we examined the effects of MEK inhibitor U0126 and PI3K inhibitor LY294002 on the growth of both SK-HEP1 shTF-Vector and -TF cells. Treatment with U0126 or/and LY294002 decreased the protein levels of EGFR, c-Myc, pERK or/and pAKT in both SK-HEP1 shTFVector and -TF cells (Figures 3B-D). However, with U0126 or LY294002 alone inhibited the growth only in SK-HEP1 shTFTF cells but not in SK-HEP1 shTF-Vector cells. After treating with the combination of U0126 and LY294002 significantly

TABLE 1 | The correlation between TF and EGFR protein expressions in HCC tissues.

\begin{tabular}{|c|c|c|c|c|c|}
\hline & & \multicolumn{3}{|c|}{ TF expression } & \multirow{2}{*}{$\begin{array}{l}P \\
r\end{array}$} \\
\hline & & High & Low & Total & \\
\hline EGFR & High & 82 & 9 & 91 & $<0.001$ \\
\hline \multirow[t]{2}{*}{ expression } & Low & 23 & 30 & 53 & 0.668 \\
\hline & & 105 & 39 & 144 & \\
\hline
\end{tabular}
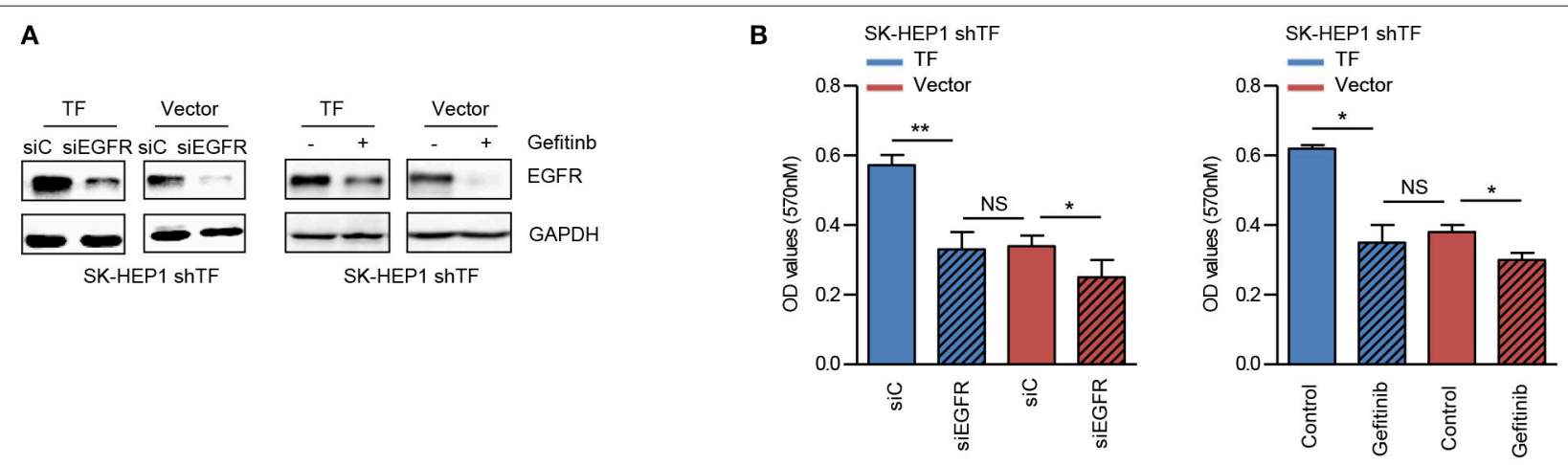

FIGURE 4 | Inhibition of EGFR suppresses TF-mediated HCC growth. SK-HEP1 shTF-Vector and SK-HEP1 shTF-TF cells were transfected with siControl or siEGFR or treated with/without gefinib at the concentration of $10 \mu \mathrm{M}$ for $24 \mathrm{~h}$. (A) Western blot and (B) MTT assay analysis of the protein expressions and cell growth. Error bars, mean \pm SD. ${ }^{*} p<0.05$ and ${ }^{* *} p<0.01$ (two-tailed Student's $t$-test B). 


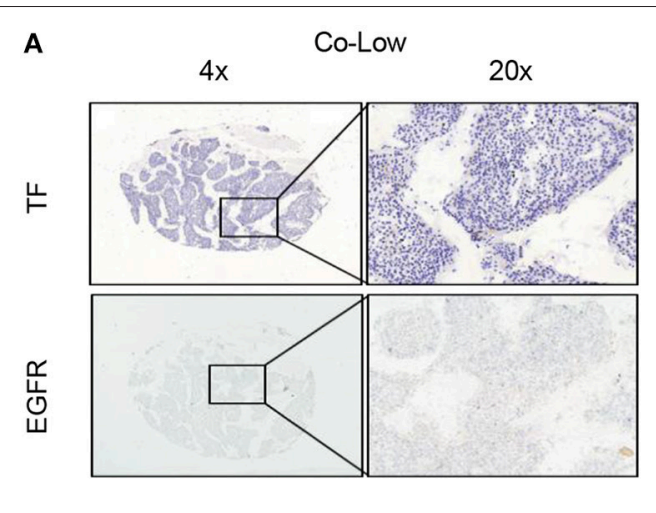

B

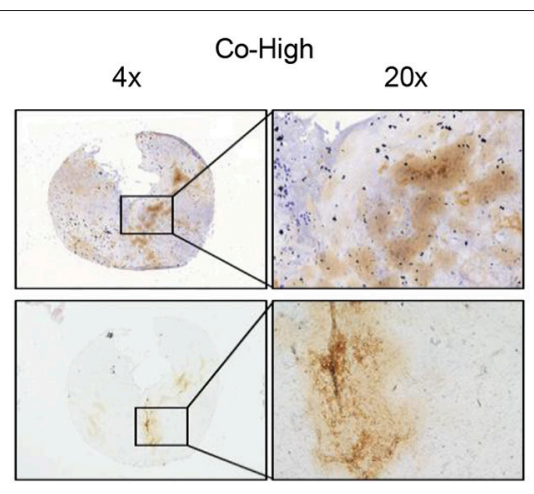

C

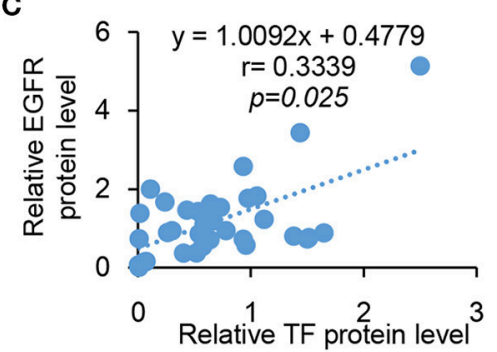

FIGURE 5 | TF protein expression is correlated with EGFR and poor HCC patient prognosis. TF and EGFR protein expressions in 144 HCC tissues were examined with IHC assay. (A) Representative images of positive and negative expression of both TF and EGFR were shown at $4 \mathrm{X}$ and $20 \mathrm{X}$ magnification. (B) Representative images of western blot analysis of TF and EGFR protein expression in the paired HCC tissues and adjacent normal tissues. (C) Spearman's rank correlation test showed the correlation between TF and EGFR protein expressions by Western blot.

inhibited the growth in both SK-HEP1 shTF-Vector and TF cells (Figure 3E). In short, these data suggest that TF promotes the growth of HCC by activating both ERK and AKT signaling pathways.

\section{Inhibition of EGFR Suppresses TF-Mediated HCC Growth}

EGFR has been identified as a key player in the development of HCC (25). To verify the role of EGFR in TF-mediated HCC growth, we examined the effects of EGFR siRNA and EGFR inhibitor gefitinib on the growth of both SK-HEP1 shTF-Vector and -TF cells. EGFR siRNA or gefitinib decreased the protein levels of EGFR in both SK-HEP1 shTF-Vector and -TF cells (Figure 4A). Furthermore, EGFR siRNA or gefitinib inhibited the growth more significantly in SK-HEP1 shTF-TF cells than in SK-HEP1 shTF-Vector cells, indicating that inhibition of EGFR suppresses TF-mediated HCC growth (Figure 4B).

\section{TF Protein Expression Is Correlated With EGFR in HCC Tissues}

Our results clearly demonstrate that EGFR is regulated by TF in cell culture. To determine whether this is also the case in tumor tissues, we compared the protein levels of TF and EGFR in human 144 HCC tissues by IHC assay. High TF and EGFR staining were present in 105 (72.9\%) and 91 (63.2\%) out of 144 HCC tissues, respectively. Results of representative tissues with co-low or cohigh staining of TF and EGFR were shown in Figure 5A. The expression of TF was highly correlated with the expression of EGFR in HCC tissues (Table $\mathbf{1}$ and Figures 5B, C).

\section{DISCUSSION}

It has been demonstrated that TF-induced tumor progression need the activation of intracellular signaling pathways, where TF cytoplasmic domain couples to proteolytic activation of the protease activated receptor (PAR) 2 and subsequently activates ERK, AKT and other signaling pathways (26). For example, TF was involved in retinoblastoma cell proliferation via activating both ERK and AKT signaling pathways (27). Knockdown of TF suppressed human lung adenocarcinoma growth in vitro and in vivo through inhibiting both ERK and AKT signaling pathways (28). Similarly, our results showed that TF promoted the growth of HCC in vitro and in vivo by activating both ERK and AKT signaling pathways. Inhibition of ERK and AKT blocked TFmediated growth of HCC. Therefore, activation of both ERK and AKT signaling pathways is indispensable for TF-promoted the growth of HCC.

EGFR is a member of ErbB/HER family of transmebrane receptor tyrosine kinases. It is activated by specific ligands resulting in the activation of multiple intracellular signaling pathways including ERK, AKT. Those signaling pathways is related to cell proliferation, migration and invasion (29-31). The gene expression of EGFR is regulated by the transcription factor c-Myc (32). In this study, we found that TF could enhance 
the expression of c-Myc and EGFR, and inhibition of ERK and AKT could block TF-induced c-Myc and EGFR upregulation. Phosphorylation of serine 62 amino acid residues by ERK prevents c-Myc protein from degradation (33). AKT stabilizes $\mathrm{c}-\mathrm{Myc}$ protein by phosphorylation and inactivation of GSK-3 $\beta$ which phosphorylated threonine 58 amino acid residues of c-Myc to promote c-Myc degradation (33).

Inhibition of EGFR with either small molecule inhibitors or specific antibodies has achieved promising results in the preclinical HCC models. In human HCC cells, gefitinib, erlotinib or cetuximab could induce growth inhibition, cell cycle arrest and apoptosis (34-36). In the orthotopic HCC models, gefitinib significantly inhibited the growth and metastasis of HCC tumors, and enhanced by the combination with cisplatin $(37,38)$. However, the outcome of targeting EGFR in HCC was modest in the clinical trials. When used as a single agent in HCC patients, erlotinib only acquired moderate effects $(39,40)$, and cetuximab showed no antitumor activity (41). Treatment failure with EGFR inhibitors in HCC patients may cause by many reasons, such as the levels and mutations of EGFR, EMT status of tumor cells, etc. (42-44). In the current study, we found that treatment with EGFR siRNA or gefitinib suppressed the growth more significantly in the TF highly expressed HCC cells, suggesting that the levels of TF in tumor cells may influence the effects of EGFR inhibitors. Furthermore, our IHC data showed that both positive ratios of TF and EGFR protein in the HCC tissue were $72.9 \%(105 / 144)$ and $63.2 \%(91 / 144)$, respectively. The expression of TF was highly correlated with the expression of EGFR in HCC tissues. Therefore, it may be valuable to investigate the relation of TF expressions and EGFR inhibitors effects in the future studies.

\section{REFERENCES}

1. Torre LA, Bray F, Siegel RL, Ferlay J, Lortet-Tieulent J, Jemal A. Global cancer statistics, 2012. CA Cancer J Clin. (2015) 65:87-108. doi: 10.3322/caac.21262

2. Chen W, Zheng R, Baade PD, Zhang S, Zeng H, Bray F, et al. Cancer statistics in China, 2015. CA Cancer J Clin. (2016) 66:115-32. doi: 10.3322/caac.21338

3. Sun T, Liu H, Ming L. Multiple roles of autophagy in the sorafenib resistance of hepatocellular carcinoma. Cell Physiol Biochem. (2017) 44:716-27. doi: 10.1159/000485285

4. Li C, Chen J, Zhang K, Feng B, Wang R, Chen L. Progress and prospects of long noncoding RNAs (lncRNAs) in hepatocellular carcinoma. Cell Physiol Biochem. (2015) 36:423-34. doi: 10.1159/0004 30109

5. Versteeg HH, Spek CA, Peppelenbosch MP, Richel DJ. Tissue factor and cancer metastasis: the role of intracellular and extracellular signaling pathways. Mol Med. (2004) 10:6-11. doi: 10.2119/2003-00047.Versteeg

6. Mackman N. Role of tissue factor in hemostasis, thrombosis, and vascular development. Arterioscler Thromb Vasc Biol. (2004) 24:1015-22. doi: 10.1161/01.ATV.0000130465.23430.74

7. Ruf W. Tissue factor and cancer. Thromb Res. (2012) 130(Suppl. 1):S84-87. doi: 10.1016/j.thromres.2012.08.285

8. Zhou Q, Huang T, Wang YF, Zhou XB, Liang LJ, Peng BG. Role of tissue factor in hepatocellular carcinoma genesis, invasion and metastasis. Chin Med J. (2011) 124:3746-51.

9. Kaido T, Oe H, Yoshikawa A, Mori A, Arii S, Imamura M. Tissue factor is a useful prognostic factor of recurrence in hepatocellular carcinoma in 5-year survivors. Hepatogastroenterology. (2005) 52:1383-7.

\section{CONCLUSIONS}

Our results provide proof-of-principle insights into a novel mechanism driven by TF on HCC growth and suggest that TF and EGFR may be potential therapeutic targets of HCC.

\section{DATA AVAILABILITY}

The datasets generated for this study are available on request to the corresponding author.

\section{AUTHOR CONTRIBUTIONS}

S-ZH, M-NW, J-RH, Z-JZ, W-JZ, Q-WJ, and YY performed experiments. H-YW, H-LJ, KW, Z-HX, M-LY, and YL collected and analyzed data. X-SH, ZS, and QZ prepared the manuscript.

\section{FUNDING}

This work was supported by funds from the National Natural Science Foundation of China Nos. 81661148049 and 81772540 (ZS), the Guangdong Natural Science Funds for Distinguished Young Scholar No. 2014A030306001 (ZS), the Guangdong Special Support Program for Young Talent No. 2015TQ01R350 (ZS), the Science and Technology Program of Guangdong Nos. 201300000187 (QZ) and 2016A050502027 (ZS), the Science and Technology Program of Guangzhou No. 201704030058 (ZS), the Science and Technology Program of Huizhou (170520181743174/2017Y229 and 180529101741637/2018Y305), and the Program Sci-tech Research Development of Guangdong Province 2014A020212717 (QZ).
10. Poon RT, Lau CP, Ho JW, Yu WC, Fan ST, Wong J. Tissue factor expression correlates with tumor angiogenesis and invasiveness in human hepatocellular carcinoma. Clin Cancer Res. (2003) 9:5339-45.

11. Yang Y, Qiu JG, Li Y, Di JM, Zhang WJ, Jiang QW, et al. Targeting ABCB1mediated tumor multidrug resistance by CRISPR/Cas9-based genome editing. Am J Transl Res. (2016) 8:3986-94.

12. Shi Z, Li Z, Li ZJ, Cheng K, Du Y, Fu H, et al. Cables1 controls p21/Cip1 protein stability by antagonizing proteasome subunit alpha type 3. Oncogene. (2015) 34:2538-45. doi: 10.1038/onc.2014.171

13. Luo Y, Jiang QW, Wu JY, Qiu JG, Zhang WJ, Mei XL, et al. Regulation of migration and invasion by toll-like receptor- 9 signaling network in prostate cancer. Oncotarget. (2015) 6:22564-74. doi: 10.18632/oncotarget.4197

14. Zhang WJ, Li Y, Wei MN, Chen Y, Qiu JG, Jiang QW, et al. Synergistic antitumor activity of regorafenib and lapatinib in preclinical models of human colorectal cancer. Cancer Lett. (2017) 386:100-9. doi: 10.1016/j.canlet.2016.11.011

15. Lv M, Qiu JG, Zhang WJ, Jiang QW, Qin WM, Yang Y, et al. Wallichinine reverses ABCB1-mediated cancer multidrug resistance. Am J Transl Res. (2016) 8:2969-80.

16. Chen X, Gong L, Ou R, Zheng Z, Chen J, Xie F, et al. Sequential combination therapy of ovarian cancer with cisplatin and gamma-secretase inhibitor MK0752. Gynecol Oncol. (2016) 140:537-44. doi: 10.1016/j.ygyno.2015.12.011

17. Lin M, Bi H, Yan Y, Huang W, Zhang G, Tang S, et al. Parthenolide suppresses non-small cell lung cancer GLC-82 cells growth via B-Raf/MAPK/Erk pathway. Oncotarget. (2017) 8:23436-47. doi: 10.18632/oncotarget.15584

18. Gong LH, Chen XX, Wang H, Jiang QW, Pan SS, Qiu JG, et al. Piperlongumine induces apoptosis and synergizes with cisplatin or paclitaxel 
in human ovarian cancer cells. Oxid Med Cell Longev. (2014) 2014:906804. doi: $10.1155 / 2014 / 906804$

19. Li P, Yang Y, Liu H, Yang AK, Di JM, Tan GM, et al. MiR-194 functions as a tumor suppressor in laryngeal squamous cell carcinoma by targeting weel. $J$ Hematol Oncol. (2017) 10:32. doi: 10.1186/s13045-017-0402-6

20. Jiang QW, Cheng KJ, Mei XL, Qiu JG, Zhang WJ, Xue YQ, et al. Synergistic anticancer effects of triptolide and celastrol, two main compounds from thunder god vine. Oncotarget. (2015) 6:32790-804. doi: 10.18632/oncotarget.5411

21. Zheng DW, Xue YQ, Li Y, Di JM, Qiu JG, Zhang WJ, et al. Volasertib suppresses the growth of human hepatocellular carcinoma in vitro and in vivo. Am J Cancer Res. (2016) 6:2476-88.

22. Mei XL, Yang Y, Zhang YJ, Li Y, Zhao JM, Qiu JG, et al. Sildenafil inhibits the growth of human colorectal cancer in vitro and in vivo. Am J Cancer Res. (2015) 5:3311-24.

23. Shi Z, Park HR, Du Y, Li Z, Cheng K, Sun SY, et al. Cables1 complex couples survival signaling to the cell death machinery. Cancer Res. (2015) 75:147-58. doi: 10.1158/0008-5472.CAN-14-0036

24. Qiu JG, Zhang YJ, Li Y, Zhao JM, Zhang WJ, Jiang QW, et al. Trametinib modulates cancer multidrug resistance by targeting $\mathrm{ABCB} 1$ transporter. Oncotarget. (2015) 6:15494-509. doi: 10.18632/oncotarget.3820

25. Berasain C, Avila MA. The EGFR signalling system in the liver: from hepatoprotection to hepatocarcinogenesis. J Gastroenterol. (2014) 49:9-23. doi: 10.1007/s00535-013-0907-X

26. Han X, Guo B, Li Y, Zhu B. Tissue factor in tumor microenvironment: a systematic review. J Hematol Oncol. (2014) 7:54. doi: 10.1186/s13045-014-0054-8

27. Lee BJ, Kim JH, Woo SH, Kim DH, Yu YS. Tissue factor is involved in retinoblastoma cell proliferation via both the akt and extracellular signal-regulated kinase pathways. Oncol Rep. (2011) 26:665-70. doi: $10.3892 /$ or.2011.1314

28. Xu C, Gui Q, Chen W, Wu L, Sun W, Zhang N, et al. Small interference RNA targeting tissue factor inhibits human lung adenocarcinoma growth in vitro and in vivo. J Exp Clin Cancer Res. (2011) 30:63. doi: 10.1186/1756-9966-30-63

29. Grant S, Qiao L, Dent P. Roles of ERBB family receptor tyrosine kinases, and downstream signaling pathways, in the control of cell growth and survival. Front Biosci. (2002) 7:d376-89. doi: 10.2741/grant

30. Normanno N, De Luca A, Bianco C, Strizzi L, Mancino M, Maiello MR, et al. Epidermal growth factor receptor (EGFR) signaling in cancer. Gene. (2006) 366:2-16. doi: 10.1016/j.gene.2005.10.018

31. Ma P, Fu Y, Chen M, Jing Y, Wu J, Li K, et al. Adaptive and acquired resistance to EGFR inhibitors converge on the MAPK pathway. Theranostics. (2016) 6:1232-43. doi: 10.7150/thno.14409

32. Perini G, Diolaiti D, Porro A, Della Valle G. In vivo transcriptional regulation of n-myc target genes is controlled by E-box methylation. Proc Natl Acad Sci USA. (2005) 102:12117-22. doi: 10.1073/pnas.0409097102

33. Sears R, Nuckolls F, Haura E, Taya Y, Tamai K, Nevins JR. Multiple ras-dependent phosphorylation pathways regulate Myc protein stability. Genes Dev. (2000) 14:2501-14. doi: 10.1101/gad.8 36800
34. Okano J, Matsumoto K, Nagahara T, Murawaki Y. Gefitinib and the modulation of the signaling pathways downstream of epidermal growth factor receptor in human liver cancer cells. J Gastroenterol. (2006) 41:166-76. doi: 10.1007/s00535-005-1736-3

35. Hopfner M, Sutter AP, Huether A, Schuppan D, Zeitz M, Scherubl H. Targeting the epidermal growth factor receptor by gefitinib for treatment of hepatocellular carcinoma. J Hepatol. (2004) 41:1008-16. doi: 10.1016/j.jhep.2004.08.024

36. Huether A, Hopfner M, Sutter AP, Schuppan D, Scherubl H. Erlotinib induces cell cycle arrest and apoptosis in hepatocellular cancer cells and enhances chemosensitivity towards cytostatics. J Hepatol. (2005) 43:661-9. doi: 10.1016/j.jhep.2005.02.040

37. Matsuo M, Sakurai H, Saiki I. ZD1839, a selective epidermal growth factor receptor tyrosine kinase inhibitor, shows antimetastatic activity using a hepatocellular carcinoma model. Mol Cancer Ther. (2003) 2:557-61.

38. Zhu BD, Yuan SJ, Zhao QC, Li X, Li Y, Lu QY. Antitumor effect of gefitinib, an epidermal growth factor receptor tyrosine kinase inhibitor, combined with cytotoxic agent on murine hepatocellular carcinoma. World J Gastroenterol. (2005) 11:1382-6. doi: 10.3748/wjg.v11.i9.1382

39. Philip PA, Mahoney MR, Allmer C, Thomas J, Pitot HC, Kim G, et al. Phase II study of erlotinib (OSI-774) in patients with advanced hepatocellular cancer. J Clin Oncol. (2005) 23:6657-63. doi: 10.1200/JCO.2005.14.696

40. Thomas MB, Chadha R, Glover K, Wang X, Morris J, Brown T, et al. Phase 2 study of erlotinib in patients with unresectable hepatocellular carcinoma. Cancer. (2007) 110:1059-67. doi: 10.1002/cncr.22886

41. Zhu AX, Stuart K, Blaszkowsky LS, Muzikansky A, Reitberg DP, Clark JW, et al. Phase 2 study of cetuximab in patients with advanced hepatocellular carcinoma. Cancer. (2007) 110:581-9. doi: 10.1002/cncr.22829

42. Lanaya H, Natarajan A, Komposch K, Li L, Amberg N, Chen L, et al. EGFR has a tumour-promoting role in liver macrophages during hepatocellular carcinoma formation. Nat Cell Biol. (2014) 16:972-7. doi: 10.1038/ncb3031

43. Su MC, Lien HC, Jeng YM. Absence of epidermal growth factor receptor exon 18-21 mutation in hepatocellular carcinoma. Cancer Lett. (2005) 224:117-21. doi: 10.1016/j.canlet.2004.10.010

44. Fuchs BC, Fujii T, Dorfman JD, Goodwin JM, Zhu AX, Lanuti M, et al. Epithelial-to-mesenchymal transition and integrin-linked kinase mediate sensitivity to epidermal growth factor receptor inhibition in human hepatoma cells. Cancer Res. (2008) 68:2391-9. doi: 10.1158/0008-5472.CAN-07-2460

Conflict of Interest Statement: The authors declare that the research was conducted in the absence of any commercial or financial relationships that could be construed as a potential conflict of interest.

Copyright @ 2019 Huang, Wei, Huang, Zhang, Zhang, Jiang, Yang, Wang, Jin, Wang, Xing, Yuan, Li, He, Shi and Zhou. This is an open-access article distributed under the terms of the Creative Commons Attribution License (CC BY). The use, distribution or reproduction in other forums is permitted, provided the original author(s) and the copyright owner(s) are credited and that the original publication in this journal is cited, in accordance with accepted academic practice. No use, distribution or reproduction is permitted which does not comply with these terms. 